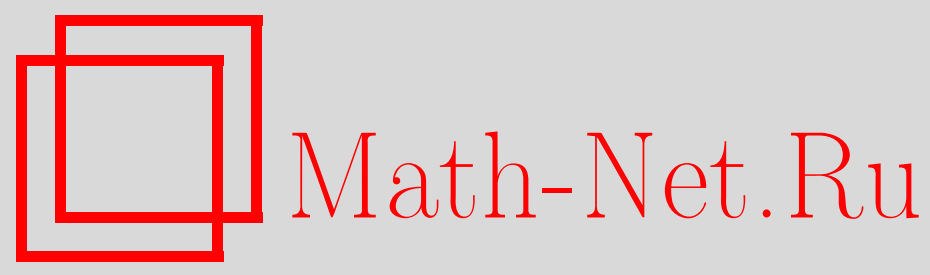

Е. Д. Лившиц, О почти наилучшем приближении кусочнополиномиальными функциями в пространстве $C[0,1], \mathrm{Ma}$ тем. заметки, 2005, том 78, выпуск 4, 629-633

DOI: https://doi.org/10.4213/mzm2625

Использование Общероссийского математического портала Math-Net.Ru подразумевает, что вы прочитали и согласны с пользовательским соглашением http://www.mathnet.ru/rus/agreement

Параметры загрузки:

IP : 54.80 .97 .219

26 апреля 2023 г., 14:32:17

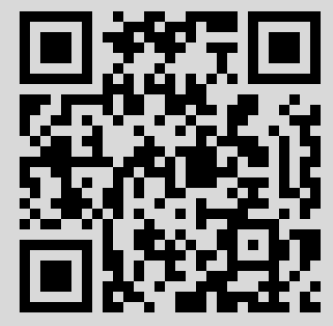




\section{О ПОЧТИ НАИЛУЧШЕМ ПРИБЛИЖЕНИИ КУСОЧНО-ПОЛИНОМИАЛЬНЫМИ ФУНКЦИЯМИ В ПРОСТРАНСТВЕ $C[0,1]$}

\section{Е. Д. Лившиц}

Введение. Пусть заданы $X=\left(X,\|\cdot\|_{X}\right)$ - действительное банахово пространство и его подмножество $M$. Оператором метрической проекиии назьвается, вообще говоря, многозначное отображение

$$
P_{M, X}: x \in X \mapsto\left\{z \in M:\|x-z\|_{X}=\rho_{X}(x, M)\right\},
$$

где $\rho_{X}(x, M)=\inf _{z \in M}\|x-z\|_{X}$. Однозначное отображение $G: X \rightarrow M$ называется мультипликативной (соответственно аддитивной) $\varepsilon$-въборкой, $\varepsilon \geqslant 0$, из $X$ в $M$, если

$$
\forall x \in X \quad\|x-G(x)\| \leqslant \rho(x, M)(1+\varepsilon) \quad(\text { соответственно }\|x-G(x)\| \leqslant \rho(x, M)+\varepsilon) .
$$

Если $G$ непрерывно, будем говорить, что $G$ - непреры вная выборка. И. Г. Царьков доказал, что для замкнутого множества $M$ сушествование для любого $\varepsilon>0$ непрерывных мультипликативных $\varepsilon$-выборок в него эквивалентно существованию для всех $\varepsilon>0$ непрерывных аддитивных $\varepsilon$-выборок (в него). Обозначим через $\mathscr{P}_{d}[a, b]$ множество алгебраических полиномов степени $\leqslant d$, определенных на $[a, b]$, а через $R_{m, n}[a, b]$ множество алгебраических рациональных дробей

$$
R_{m, n}[a, b]=\left\{\frac{P}{Q} ; P \in \mathscr{P}_{m}[a, b] ; Q \in \mathscr{P}_{n}[a, b] ; Q(t) \neq 0 \text { при } \forall t \in[a, b]\right\} .
$$

В 1859 году Чебышев [1] отметил, что для любой функции $f \in C[0,1]$ и фиксированных $d, m, n \geqslant 0$ множества $P_{\mathscr{P}_{d}[0,1], C[0,1]}(f)$ и $P_{R_{m, n}[0,1], C[0,1]}(f)$ состоят из единственной функции. Кирхбергер [2] доказал, что для любого $d \geqslant 0$ (однозначное) отображение $P_{\mathscr{P}}[0,1], C[0,1](\cdot)$ непрерывно. С другой стороны, Мэли и Вицгол [3] показали, что при $m, n \geqslant 1$ метрическая проекция $P_{R_{m, n}[0,1], C[0,1]}(\cdot)$ разрывна. Тем не менее, в 1988 году Конягин [4] установил, что для любого $\varepsilon>0$ существует непрерывная аддитивная $\varepsilon$-выборка в множество $R_{m, n}$. Мультипликативные выборки в множества $R_{m, n}$ в $C[0,1]$, обладающие большей устойчивостью, изучались Рютиньтм [5], [6].

Наряду с рациональньми дробями в качестве аппроксимирующего аппарата в теории приближений также используются кусочно-полиномиальные функции с нефиксированными узлами

$$
\begin{aligned}
S_{n}^{d}[a, b]=\{f \in C[a, b]: & \exists a=x_{0}<x_{1}<\cdots<x_{n}=b, \\
& \left.\left.f\right|_{\left[x_{k-1}, x_{k}\right]} \in \mathscr{P}_{d}\left[x_{k-1}, x_{k}\right], 1 \leqslant k \leqslant n\right\} .
\end{aligned}
$$

Девор, Ховард и Мичелли [7] построили непрерьвные мультипликативные $\varepsilon$-выборки из соболевских шаров в $S_{n}^{1}[0,1]$. В работе [8] изучались выборки в $S_{n}^{1}[0,1]$ из пространства $C[0,1]$. Было доказано, что 0-выборка (выборка из метрической проекции) в $S_{n}[0,1]$ существует только для $n=1,2$, но для любого $\varepsilon>0$ и $n \geqslant 1$ существует непрерьвная аддитивная (и, следовательно, мультипликативная) $\varepsilon$-выборка в $S_{n}^{1}$. В этой работе изучаются непрерывные мультипликативные выборки из пространства $C[0,1]$ в $S_{n}^{d}[0,1], d>1$.

ТеОРема 1. Для любых $\varepsilon>0, n \geqslant 1, d \geqslant 1$ существует непрерывная мультипликативная $(1+\varepsilon)$-виборка из $C[0,1]$ в $S_{n}^{d}[0,1]$.

Работа выполнена при финансовой поддержке Российского фонда фундаментальных исследований, грант № 05-01-00062. 
Теорема 2. Для любъх $\varepsilon>0, n \geqslant 2, d \geqslant 2$ не существует непрерывной мультипликативной $(1-\varepsilon)$-выборки из $C[0,1]$ в $S_{n}^{d}[0,1]$.

Доказательство теоремы 1 . Без ограничения общности можно считать, что $0<\varepsilon<1$. Через $\|\cdot\|_{[a, b]}$ будем обозначать норму в $C[a, b]$. Для краткости мы будем писать $S_{n}^{d}$ и $\|\cdot\|$ вместо $S_{n}^{d}[0,1]$ и $\|\cdot\|_{[0,1]}$ соответственно. Пусть $s \in S_{n}^{d}$. Будем говорить, что $t \in H_{1}(s)$, если существует такое разбиение $0=x_{0}<x_{1}=t<\cdots<x_{n-1}<x_{n}=1$, что

$$
\left.s\right|_{\left[x_{k}, x_{k+1}\right]} \in \mathscr{P}_{d}\left[x_{k}, x_{k+1}\right], \quad 0 \leqslant k \leqslant n-1 .
$$

ЛЕмма 1. Для любого $k, 2 \leqslant k \leqslant n, u \gamma>0$ существует такое непрерывное отображение $F_{k, \gamma}: C[0,1] \backslash S_{k}^{d} \rightarrow(0,1)$, что для каждой $f \in C[0,1] \backslash S_{k}^{d}$ найдутся $p \in \mathscr{P}_{d}\left[0, F_{k, \gamma}(f)\right]$ $u s \in S_{k-1}^{d}\left[F_{k, \gamma}(f), 1\right]$, удовлетворяющие неравенствам

$$
\|f-p\|_{\left[0, F_{k, \gamma}(f)\right]} \leqslant \rho\left(f, S_{n}^{d}\right)(1+\gamma), \quad\|f-s\|_{\left[F_{k, \gamma}(f), 1\right]} \leqslant \rho\left(f, S_{n}^{d}\right)(1+\gamma) .
$$

ДоКАЗАТЕЛЬСтво. Для $k, 2 \leqslant k \leqslant n$, рассмотрим функцию $T: C[0,1] \times(0,1) \rightarrow \mathbb{R}$, заданную соотношением

$$
T(f, t)=\sup _{s \in S_{k}^{d}, t \in H_{1}(s)}\left(\rho\left(f, S_{k}^{d}\right)\left(1+\frac{\gamma}{2}\right)-\|f-s\|\right)_{+} .
$$

Докажем, что требуемое отображение можно задать формулой

$$
F_{k, \gamma}(f)=\frac{\int_{0}^{1} t T(f, t) d t}{\int_{0}^{1} T(f, t) d t}
$$

Нетрудно видеть, что $T(\cdot, \cdot)$ непрерьвна на области определения и, если $f \notin S_{k}^{d}$, то в силу замкнутости $S_{k}^{d}$ выполняется $\rho\left(f, S_{k}^{d}\right)>0$ и существует $t \in(0,1)$, для которого $T(f, t)>0$. Отсюда следует непрерывность $F_{k, \gamma}(\cdot)$ на области определения. Из определений (1) и (2) следует, что для любой $f \in C[0,1] \backslash S_{k}^{d}$ найдутся $t_{1}, t_{2}, 0<t_{1}<F_{k, \gamma}(f)<t_{2}<1$, и $s_{1}, s_{2} \in S_{k}^{d}$, для которых $t_{i} \in H_{1}\left(s_{i}\right)$ и $\left\|f-s_{i}\right\| \leqslant \rho\left(f, s_{k}^{d}\right)(1+\gamma), i=1,2$. Тогда можно положить $p=\left.s_{2}\right|_{\left[0, F_{k, \gamma}(f)\right]}$ и $s=\left.s_{1}\right|_{\left[F_{k, \gamma}(f), 1\right]}$.

Построение мультипликативной $(1+\varepsilon)$-выборки $G(\cdot)$. Обозначим через $L_{[\alpha, \beta]}^{[\gamma, \delta]}$ линейное отображение, переводящее отрезок $[\alpha, \beta]$ в $[\gamma, \delta]$ :

$$
L_{[\alpha, \beta]}^{[\gamma, \delta]}(t)=\frac{\delta-\gamma}{\beta-\alpha}(t-\alpha)+\gamma
$$

Если $f \in S_{n}^{d}$, то положим $G(f)=f$, в противном случае положим $x_{0}^{a v}=x_{0}^{a v}(f)=0, x_{n}^{a v}=$ $x_{n}^{a v}(f)=1$ и последовательно определим $0<x_{1}^{a v}<x_{2}^{a v}<\cdots<x_{n-1}^{a v}<1$ :

$$
x_{k+1}^{a v}=x_{k+1}^{a v}(f)=L_{[0,1]}^{\left[x_{k}^{a v}, 1\right]}\left(F_{n-k, \varepsilon /(4 n)}\left(f \circ L_{[0,1]}^{\left[x_{k}^{a v}, 1\right]}\right)\right), \quad k=0,1, \ldots, n-2 .
$$

Рассмотрим полиномы

$$
p_{k}=p_{k}(f)=P_{\mathscr{P}_{d}[0,1], C[0,1]}\left(f \circ L_{[0,1]}^{\left[x_{k}^{a v}, x_{k+1}^{a v}\right]}\right), \quad 0 \leqslant k \leqslant n-1,
$$

линейные функции

$$
l_{k}(t)=\frac{1}{2}\left(p_{k-1}(1)-p_{k}(0)+t\left(-p_{k-1}(1)+p_{k}(0)-p_{k}(1)+p_{k+1}(0)\right)\right)
$$


и полиномы

$$
\widetilde{p}_{k}=\widetilde{p}_{k}(f)=p_{k}+l_{k}, \quad 0 \leqslant k \leqslant n-1,
$$

считая $p_{-1}(1)=p_{0}(0)$ и $p_{n}(0)=p_{n-1}(1)$. Ясно, что

$$
\widetilde{p}_{k}(1)=\widetilde{p}_{k+1}(0)=\frac{1}{2}\left(p_{k}(1)+p_{k+1}(0)\right) \text {. }
$$

Это делает корректным определение $G(f) \in S_{n}^{d}$ посредством равенств

$$
\left.G(f)\right|_{\left[x_{k}^{a v}, x_{k+1}^{a v}\right]} \equiv \widetilde{p}_{k} \circ L_{\left[x_{k}^{a v}, x_{k+1}^{a v}\right]}^{[0,1]}, \quad 0 \leqslant k \leqslant n-1 .
$$

В силу леммы 1 , определения (3) и неравенства $0<\varepsilon<1$ имеем для $0 \leqslant k \leqslant n-1$

$$
\begin{gathered}
\left\|f \circ L_{[0,1]}^{\left[x_{k}^{a v}, x_{k+1}^{a v}\right]}-p_{k}\right\| \leqslant \rho\left(f, S_{n}^{d}\right)\left(1+\frac{\varepsilon}{4 n}\right)^{n} \leqslant \rho\left(f, S_{n}^{d}\right)\left(1+\frac{\varepsilon}{2}\right), \\
\left|l_{k}(0)\right|=\frac{1}{2}\left|p_{k-1}(1)-p_{k}(0)\right| \leqslant \frac{1}{2}\left(\left|p_{k-1}(1)-f\left(x_{k}^{a v}\right)\right|+\left|f\left(x_{k}^{a v}\right)-p_{k}(0)\right|\right) \\
=\frac{1}{2}\left(\left|p_{k-1}(1)-f \circ L_{[0,1]}^{\left[x_{k-1}^{a v}, x_{k}^{a v}\right]}(1)\right|+\left|f \circ L_{[0,1]}^{\left[x_{k}^{a v}, x_{k+1}^{a v}\right]}(0)-p_{k}(0)\right|\right) \leqslant \rho\left(f, S_{n}^{d}\right)\left(1+\frac{\varepsilon}{2}\right) .
\end{gathered}
$$

Точно так же проверяется, что

$$
\left|l_{k}(1)\right| \leqslant \rho\left(f, S_{n}^{d}\right)\left(1+\frac{\varepsilon}{2}\right)
$$

и в силу линейности

$$
\left\|l_{k}\right\| \leqslant \rho\left(f, S_{n}^{d}\right)\left(1+\frac{\varepsilon}{2}\right)
$$

Следовательно, по неравенству треугольника,

$$
\left\|f \circ L_{[0,1]}^{\left[x_{k}^{a v}, x_{k+1}^{a v}\right]}-\widetilde{p}_{k}\right\| \leqslant \rho\left(f, S_{n}^{d}\right)(2+\varepsilon) .
$$

Отсюда непосредственно вытекает, что $\|f-G(f)\| \leqslant \rho\left(f, S_{n}^{d}\right)(1+1+\varepsilon)$. Поэтому отображение $G(\cdot)$ является мультипликативной $(1+\varepsilon)$-выборкой.

Непрерывность выборки $G(\cdot)$. Докажем непрерьвность $G(\cdot)$ в произвольной точке $f_{0}$. Если $f_{0} \in S_{n}^{d}$, то $G\left(f_{0}\right)=f_{0}$. Пусть $f \in C[0,1]$ и $\left\|f-f_{0}\right\|<\delta$. В силу того, что $G(\cdot)$ является $(1+\varepsilon)$-выборкой, имеем

$$
\|f-G(f)\| \leqslant \rho\left(f, S_{n}^{d}\right)(2+\varepsilon) \leqslant\left\|f-G\left(f_{0}\right)\right\|(2+\varepsilon)=\left\|f-f_{0}\right\|(2+\varepsilon)<\delta(2+\varepsilon) .
$$

Следовательно,

$$
\left\|G(f)-G\left(f_{0}\right)\right\| \leqslant\|G(f)-f\|+\left\|f-f_{0}\right\|+\left\|f_{0}-G\left(f_{0}\right)\right\| \leqslant \delta(3+\varepsilon) \leqslant 4 \delta,
$$

что доказывает непрерывность $G(\cdot)$ в точке $f_{0} \in S_{n}^{d}$.

Пусть $f_{0} \notin S_{n}^{d}$ и $\delta>0$. В силу непрерьвности метрической проекции на $\mathscr{P}_{d}[0,1]$ существует такое $\eta>0$, что для всех $k, 0 \leqslant k \leqslant n-1$, и $\tilde{f} \in C[0,1],\left\|\tilde{f}-f_{0} \circ L_{[0,1]}^{\left[x_{k}^{a v}\left(f_{0}\right), x_{k+1}^{a v}\left(f_{0}\right)\right]}\right\|<\eta$, справедливо неравенство

$$
\left\|P_{\mathscr{P}_{d}[0,1], C[0,1]}\left(f_{0} \circ L_{[0,1]}^{\left[x_{k}^{a v}\left(f_{0}\right), x_{k+1}^{a v}\left(f_{0}\right)\right]}\right)-P_{\mathscr{P}_{d}[0,1], C[0,1]}(\widetilde{f})\right\|<\frac{\delta}{4} .
$$

Существует $\tau>0$ такое, что для любых $t_{1}, t_{2} \in[0,1],\left|t_{1}-t_{2}\right|<\tau$, выполняется

$$
\left|G\left(f_{0}\right)\left(t_{1}\right)-G\left(f_{0}\right)\left(t_{2}\right)\right|<\frac{\delta}{2} \text {. }
$$


В силу непрерьвности $F_{k, \gamma}(\cdot)$ (лемма 1 ) найдется $\lambda>0$ такое, что для всех $f \in C[0,1],\left\|f-f_{0}\right\|$ $<\lambda, 0 \leqslant k \leqslant n-1, t \in[0,1]$ выполняются неравенства

$$
\begin{gathered}
\left|L_{[0,1]}^{\left[x_{k}^{a v}(f), x_{k+1}^{a v}(f)\right]}(t)-L_{[0,1]}^{\left[x_{k}^{a v}\left(f_{0}\right), x_{k+1}^{a v}\left(f_{0}\right)\right]}(t)\right|<\tau, \\
\left\|f \circ L_{[0,1]}^{\left[x_{k}^{a v}(f), x_{k+1}^{a v}(f)\right]}-f_{0} \circ L_{[0,1]}^{\left[x_{k}^{a v}\left(f_{0}\right), x_{k+1}^{a v}\left(f_{0}\right)\right]}\right\|<\eta .
\end{gathered}
$$

Следовательно, согласно $(4)\left\|\widetilde{p}_{k}(f)-\widetilde{p}_{k}\left(f_{0}\right)\right\|<\delta / 2$. Тогда по определению выборки $G(\cdot)$ для любого $t \in[0,1]$ вьполняется

$$
\left|G(f)\left(L_{[0,1]}^{\left[x_{k}^{a v}(f), x_{k+1}^{a v}(f)\right]}(t)\right)-G\left(f_{0}\right)\left(L_{[0,1]}^{\left[x_{k}^{a v}\left(f_{0}\right), x_{k+1}^{a v}\left(f_{0}\right)\right]}(t)\right)\right|<\frac{\delta}{2} .
$$

Используя (5) и (6), получим, что

$$
\begin{aligned}
& \left|G(f)\left(L_{[0,1]}^{\left[x_{k}^{a v}(f), x_{k+1}^{a v}(f)\right]}(t)\right)-G\left(f_{0}\right)\left(L_{[0,1]}^{\left[x_{k}^{a v}(f), x_{k+1}^{a v}(f)\right]}(t)\right)\right| \\
& \leqslant\left|G(f)\left(L_{[0,1]}^{\left[x_{k}^{a v}(f), x_{k+1}^{a v}(f)\right]}(t)\right)-G\left(f_{0}\right)\left(L_{[0,1]}^{\left[x_{k}^{a v}\left(f_{0}\right), x_{k+1}^{a v}\left(f_{0}\right)\right]}(t)\right)\right| \\
& \quad+\left|G\left(f_{0}\right)\left(L_{[0,1]}^{\left[x_{k}^{a v}\left(f_{0}\right), x_{k+1}^{a v}\left(f_{0}\right)\right]}(t)\right)-G\left(f_{0}\right)\left(L_{[0,1]}^{\left[x_{k}^{a v}(f), x_{k+1}^{a v}(f)\right]}(t)\right)\right|<\delta .
\end{aligned}
$$

Отсюда в силу произвольности $k$ и $t$ вытекает, что $\left\|G(f)-G\left(f_{0}\right)\right\|<\delta$, и непрерывность выборки $G(\cdot)$ в точке $f_{0}$ доказана.

Доказательство теоремы 2. Для простоты изложения мы проведем доказательство теоремы в случае $n=2$, а потом укажем, как перейти к общему случаю. Мы будем использовать слегка модифицированный вариант леммы 6.1 из работы [8] (его доказательство отличается от доказательства леммы 6.1 лишь незначительньми изменениями).

ЛЕмма 2. Пусть заданы банахово пространство $X$, его подмножсества $M, V_{0}, V_{1} \subset X$, число $\gamma>0$ u $x \in X$. Если

$$
\begin{gathered}
d\left(V_{0}, V_{1}\right)=\inf _{x_{0} \in V_{0}, x_{1} \in V_{1}}\left\|x_{0}-x_{1}\right\|>0, \\
P_{M, \gamma, X}(x)=\{y \in M:\|x-y\| \leqslant \rho(x, M)(1+\gamma)\} \subset V_{0} \cup V_{1}, \\
\exists y_{i} \in V_{i}: \quad y_{i} \in P_{M, X}(x), \quad i=0,1,
\end{gathered}
$$

то не существует непрерывной мультипликативной $\gamma$-въборки из $X$ в $M$.

Пусть $\Delta, 0<\Delta<1 / 4$, и $N \in \mathbb{N}$ - фиксированные числа, которые будут заданы позднее. Пусть $p_{0}(t)=0, p_{1}(t)=-\left(2 / \Delta^{2}\right)(t-1 / 2+\Delta)(t-1 / 2-\Delta)$. Рассмотрим $y_{0}, y_{1} \in S_{2}^{d}$ :

$$
y_{0}(t)=\left\{\begin{array}{l}
p_{0}(t), 0 \leqslant t \leqslant \frac{1}{2}+\Delta, \\
p_{1}(t), \frac{1}{2}+\Delta \leqslant t \leqslant 1,
\end{array} \quad y_{1}(t)=\left\{\begin{array}{l}
p_{0}(t), 0 \leqslant t \leqslant \frac{1}{2}-\Delta, \\
p_{1}(t), \frac{1}{2}-\Delta \leqslant t \leqslant 1 .
\end{array}\right.\right.
$$

Рассмотрим функцию $f_{\Delta, N} \in C[0,1]$, заданную соотношениями

$$
\begin{gathered}
f_{\Delta, N}\left(\frac{j}{N}\right)=\left\{\begin{array}{l}
\max \left(y_{0}\left(\frac{j}{N}\right), y_{1}\left(\frac{j}{N}\right)\right)-1, j \text { четное } \\
\min \left(y_{0}\left(\frac{j}{N}\right), y_{1}\left(\frac{j}{N}\right)\right)+1, j \text { нечетное }
\end{array}\right. \\
\left.f_{\Delta, N}\right|_{[j / N,(j+1) / N]} \in \mathscr{P}_{1}\left[\frac{j}{N}, \frac{j+1}{N}\right] .
\end{gathered}
$$


В первом определении $0 \leqslant j \leqslant N$, во втором $-0 \leqslant j \leqslant N-1$. Пусть

$$
V_{0}=\left\{y \in S_{2}^{d}: y\left(\frac{1}{2}\right) \leqslant 1-\frac{\varepsilon}{2}\right\}, \quad V_{1}=\left\{y \in S_{2}^{d}: y\left(\frac{1}{2}\right) \geqslant 1+\frac{\varepsilon}{2}\right\} .
$$

Тогда $y_{i} \in V_{i}, i=0,1$, и $d\left(V_{0}, V_{1}\right) \geqslant \varepsilon>0$. Из равенства $\left\|y_{0}-y_{1}\right\|=2$, определения (7) и теоремы Чебышева об альтернансе следует, что для достаточно больших $N$ выполняется $\rho\left(f_{\Delta, N}, S_{2}^{d}\right)=1$ и, следовательно, $y_{0}, y_{1} \in P_{S_{2}^{d}, C[0,1]}\left(f_{\Delta, N}\right)$. Докажем, что при достаточно большом $N$ и малом $\Delta$ справедливо $P_{S_{2}^{d}, 1-\varepsilon, C[0,1]}\left(f_{\Delta, N}\right) \subset V_{0} \cup V_{1}$. Действительно, пусть $y \in P_{S_{2}^{d}, 1-\varepsilon, C[0,1]}\left(f_{\Delta, N}\right)$ и $t \in H_{1}(s)$. Если $t \geqslant 1 / 2$, то $\left.y\right|_{[0, t]} \in \mathscr{P}_{d}[0, t]$, и в силу неравенства $\left\|y-f_{\Delta, N}\right\| \leqslant 1(1+1-\varepsilon)$ выполняются следующие неравенства:

$$
\begin{gathered}
y\left(\frac{j}{N}\right) \leqslant-1+(2-\varepsilon)=1-\varepsilon \quad \text { для четных } j, \quad \frac{j}{N} \leqslant \frac{1}{2}-\Delta, \\
y\left(\frac{j}{N}\right) \geqslant-1+\varepsilon \quad \text { для нечетных } j, \quad \frac{j}{N} \leqslant \frac{1}{2}-\Delta .
\end{gathered}
$$

Ясно, что если $\Delta$ достаточно мало, а $N$ достаточно велико, то для $t, 0 \leqslant t \leqslant 1 / 2$, выполняется неравенство $|y(t)| \leqslant 1-\varepsilon / 2$ и, следовательно, $y \in V_{0}$. Если $t \leqslant 1 / 2$, то аналогичным образом можно показать, что $y \in V_{1}$. Таким образом, мы можем воспользоваться леммой 2 для $M=S_{2}^{d}$, $X=C[0,1], x=f_{\Delta, N}$ и $\gamma=1-\varepsilon$.

В случае большего числа звеньев надо реализовать предложенную конструкцию на отрезке $[0,1 / 2]$ и доопределить $y_{0}(\cdot)$ и $y_{1}(\cdot)$ на отрезке $[1 / 2,1]$ одинаковой кусочно-полиномиальнй функцией с соответствующим числом звеньев и достаточно большим колебанием.

\section{СПИСОК ЦИТИРОВАННОЙ ЛИТЕРАТУРЫ}

1. Чебышев П. Л. // Собр. сочинений. Т. 2. М.-Л., 1946-1951. С. 151-235. 2. Kirchberger P. Über Tschebyschefsche Annäherungsmethoden. Inauguraldissertation. Göttingen, 1902. 3. Maehly H., Witzgall Ch. // Numer. Math. 1960. V. 2. №5. P. 293-307. 4. Конягин С. В. // Матем. заметки. 1988. Т. 44. № 3. С. 404. 5. Рютин К. С. // Фундамент. и прикл. матем. 2000. Т. 6. № 4. С. 1205-1220. 6. Рютин К. С. // Матем. заметки. 2002. Т. 71. № 2. С. 261-270. 7. DeVore R., Howard R., Micchelli Ch. // Manuscripta Math. 1989. V. 63. № 4. P. 469-478. 8. Лившиц Е. Д. // Изв. РАН. Сер. матем. 2003. Т. 67. № 1. С. 99-130. 